\title{
Behavioral Sciences and Drug Discovery
}

\author{
Salvatore Enna, Professor of Physiology and Pharmacology; Associate Dean \\ for Research and Graduate Education, University of Kansas Medical Center
}

$\mathrm{M}$ any drugs discovered by our prehistoric ancestors are those affecting behavior. As this was not the result of an organized search for such agents, it reflects the fact that humans notice when consumption of a product alters their sensorium. In some cases the effect was perceived as beneficial, whereas in others it was dysphoric. Word of these discoveries was passed on for generations. Over the millennia, these products were employed for recreational or religious purposes, as poisons, for inducing sleep, or for enhancing endurance.

Defining the behavioral effects of chemical substances remains an important component in the modern era ( 1850 to the present) of drug discovery. Typically agents are sought that provoke desirable, or diminish undesirable, behaviors. Behavioral test are employed to determine whether a drug candidate unintentionally modifies central nervous system function, with such an action often being considered a side effect or toxicity.

Given the ongoing need to define the behavioral consequences of potential pharmaceuticals and environmental agents, there is a critical need for research and training in the behavioral sciences. Unfortunately, support for such programs has waned. This is due to a flagging interest in the field on the part of funding agencies, and a decline in the hiring of behavioral scientists by the pharmaceutical industry. As detailed in several publications (Enna and Williams, 2009 a \& b), including an earlier Merrill Series report (Enna, 2008), this reflects a general shift away from in vivo animal experimentation to in vitro assays for identifying and characterizing drug candidates. Provided below is a brief overview of how the behavioral sciences have historically played a major role in identifying important drugs and drug classes, the attempts made to develop new assays to substitute for detailed behavioral analyses, and efforts now being made to resurrect the behavioral sciences as a critical component of the drug discovery process.

\section{Drugs and Behavior}

In foraging for food, ancient humans would sometimes stumble upon plants or animals that provided something more than nutritional value. Occasionally, poisonous substances would be consumed, leading to illness or death. In other instances, constituents were ingested that had beneficial properties, such as anti-inflammatory, analgesic, or antimicrobial activity. Such effects would be noted only if the individual was consciously suffering from a condi- 
tion that was moderated by the product. However, the medicinal value might be overlooked if a significant amount of time elapsed between its consumption and the therapeutic effect, as would be the case with antibiotics, making it less likely the person would relate the positive response to the ingestion of a particular foodstuff. It might also be missed if days or weeks of continuous administration were needed for a beneficial response. Because the human subject was not searching specifically for such agents, centuries of consumption might pass before therapeutic benefits were noted by a sufficient number of individuals to warrant the routine collection of a particular plant or animal species for such purposes.

Unlike agents used for treating a somatic illness or infectious disease, the effects of a psychoactive compound are often noticed soon after its consumption, whether or not the individual is suffering from a medical condition. Thus, psychedelics, central nervous system stimulants, sedatives, and convulsants would be readily appreciated by the consumer, and possibly by those around him, because the substance could affect behavior. Some agents still in use today that were discovered in the pre-modern era include morphine, psilocybin and other hallucinogens, the central nervous system stimulants cocaine, caffeine, and ephedrine, and ethanol, a sedative (Table 1). While the discovery of these substances did not result from well-designed behavioral studies, their early identification and exploita- tion by individuals who were scientifically unsophisticated illustrates the value of characterizing behavioral effects in identifying novel entities for treating central nervous system disorders, and for predicting certain side effects.

The serendipitous discovery of useful, centrally-active drugs did not end with the pre-modern era. In fact, a significant number, if not a majority, of centrally-active agents, and their chemical derivatives, that are used today were discovered empirically. Examples of psychotherapeutics discovered by accident in the modern era are iproniazide and imipramine, antidepressants that were originally developed as an antitubercular agent and an antihistamine, respectively, and chlorpromazine, an antipsychotic that was initially tested as

Table 1

\begin{tabular}{cl}
\hline \multicolumn{3}{c}{ Neuroactive Drugs Discovered Empirically } \\
\hline & Morphine \\
Pre-Modern & Psilocyban \\
Era & Cocaine \\
& Ephedrine \\
& Iproniazide \\
Modern & Chlorpromazine \\
Era & Carbamazepine \\
& Valproic acid \\
& Chlordiazepoxide \\
& Modafinil \\
\hline
\end{tabular}

an adjunct for general anesthesia. The therapeutic uses of carbamazepine, an anticonvulsant and an analgesic, valproic acid, an anticonvulsant and treatment for bipolar disorder, chlordiazepoxide, an anxiolytic and sedative, and modafinil, a psychostimulant, were all discovered empirically (Table 1).

These examples, which are only a partial list of such drugs, illustrate how 
keen behavioral observations made by astute clinicians and bench scientists opened new avenues for treating mental illness and other central nervous system disorders. For example, prior to the discovery of iproniazide and chlorpromazine there were no drugs that could be used specifically to treat major depression or to alleviate symptoms of schizophrenia. Chlordiazepoxide was a seminal discovery in that it revealed a chemical class, the benzodiazepines, that could, at the proper dose, relieve anxiety without causing profound sedation. Likewise, modafinil revolutionized the treatment of narcolepsy by maintaining alertness while, unlike amphetamine, not profoundly activating central nervous system activity.

Discoveries such as these spurred training in behavioral research. It was appreciated that such expertise was needed to understand and categorize normal and abnormal human behaviors so they could be properly studied in a clinical setting. It was also important for behavioral scientists to develop laboratory animal models of central nervous system disorders that could be used in drug discovery programs aimed at identifying new chemical leads. While progress was made in achieving these goals, much remains to be done. In particular, there are serious questions about the utility of animal models of major depression and psychosis in predicting the clinical efficacy of drug candidates. As many of these tests are based on behaviors induced by agents used to treat these conditions, it is argued that they are actually models of drug action, rather than of the clinical condition itself. For this reason, a drug candidate that dis- plays possible therapeutic efficacy when examined in these models will, more than likely, have a cellular mechanism of action similar to the established agent, lessening its therapeutic impact. Accordingly, it is difficult to use such tests if the aim is to identify truly novel drugs for treating these conditions. What is needed, therefore, is additional information on the underlying neuropathology so that it can be induced, and its behavioral manifestations catalogued, in laboratory animals. As the resultant behaviors would have face validity, their modification could possibly be used to predict therapeutic potential regardless of the mechanism of action of the test agent. Until such information is accumulated, those interested in drug discovery must continue to rely on the observations of experienced behavioral scientists and clinicians for identifying new psychotherapeutics.

The importance of serendipity in the discovery of centrally-active agents has been acknowledged in the past by including a set of behavioral tests in early phases of the drug discovery program. One such battery was the Irwin test. Inclusion of a general behavioral screen left open the possibility that a neuroscientist or pharmacologist would note an unusual behavioral response early in the drug discovery process. If the compound-induced behavioral modification was perceived to have therapeutic potential, additional work could be undertaken to characterize the effect more fully to predict its clinical value. In this way novel, unanticipated, central nervous system effects would be noted and exploited for therapeutic benefit, as was done in a more haphazard manner in the 
pre-modern and early modern eras of drug discovery.

Decline of Behavioral Research in

\section{Drug Discovery}

As detailed extensively elsewhere (Enna, 2008; Enna and Williams, 2009 a $\& b)$, there was a significant shift in the design of drug discovery programs in the latter part of the $20^{\text {th }}$ century. Until that time the major emphasis was generally on first identifying chemicals that displayed some type of therapeutic potential in intact animals or organ system tests, after which studies were performed to fully characterize their potential therapeutic efficacy, safety and mechanism of action. Of these various factors, mechanism of action was least important because then, as now, an understanding of mechanism is unnecessary for regulatory approval for human use, or for exploiting such an agent for clinical benefit. What is critical is that the chemical be shown to be safe and effective in humans.

This approach to drug discovery began to change in the 1980 's, with initial tests being more focused on identifying chemicals that interact selectively, if not specifically, with known or suspected drug targets in the body. It was only after such compounds were identified using in vitro tests, that in vivo assays were performed to establish efficacy and safety in animal models of disorders thought to be related to the target site. Drug targets include enzymes, neurotransmitter and hormone receptors, ion channels, and transcription factors. This change in the approach to drug discovery from initially identifying compounds that display potential therapeutic activity in animals, to first designing selective ligands that interact with sites thought to be associated with the disorder of interest, was driven by several factors. Among these was the mapping of the human genome, which led many to believe it was just a matter of time before the genetic defects for many disorders were identified, thereby revealing the protein target that needed to be manipulated pharmacologically for therapeutic gain. It was also appreciated that in vitro screens of chemical libraries were much faster, more efficient, and less costly than labor intensive in vivo assays, such as those associated with behavioral tests. In addition, in vitro assays more readily lend themselves to automation, thereby speeding up the screening process and making it possible to test many more agents, in much smaller amounts, than in vivo assays. Automated tests were also appealing as they require fewer skilled scientists for execution and data analysis.

Automated in vivo screens, such as SmartCube and Pattern Array, were developed in an attempt to simplify and speed the assessment of test agents on animal behavior. These assay systems are capable of measuring a host of mouse behaviors in a short period of time and with little human oversight. By comparing quantified behavioral patterns between control animals, those administered a test agent, and archival data on the behavioral response to know centrally-active drugs, it is believed that predictions could be made about the behavioral effects of the drug candidate. While such tests can be an important component of a drug discovery program, they are not likely to detect an unusual response that might be noted by 
an observant and trained behavioral scientist.

As a result of this shift in research emphasis, the number of scientists trained in organ system and behavioral pharmacology declined. This was accelerated by a number of related developments, but was driven primarily by stagnation, if not an absolute decline, in federal funding for research involving vertebrate animals (Table 2).

Because federal funding determines to a great extent the nature and direction of academic research, investigators became more focused on testing hypotheses relating to molecular and bio-chemical mechanisms than organ systems biology and the behavioral sciences. Besides taking advantage of the great strides being made in molecular biology, this shift away from in vivo assays had practical consequences in that it enhanced competitiveness for funding given the growing bias against such studies. As the number of funded behavioral scientists declined, fewer students pursued graduate work in the area. Over time, the cadre of faculty qualified to conduct research and to serve as mentors in the behavioral sciences diminished as well, both through natural attrition and the reluctance of institutions to hire such individuals because of the challenges they faced in garnering grant support (Table 2). This downward spiral ultimately lead to a shortage of scientists capable of conducting well-designed, and appropriately interpreted, in vivo laboratory animal studies in general, and behavioral experiments in particular. Some argue it is no coincidence that the loss of this expertise coincided with a significant decline in the identification of new drug candidates, especially those for the treatment of neurological and psychiatric disorders (Enna and Williams, 2009a).

\section{Renaissance}

Over the past decade there has been a renewed appreciation of the importance of whole animal studies in biomedical research. This was spurred in part by the growing lag time between discovery and clinical use of new drugs

\section{Table 2}

Consequences for Behavioral Sciences

- Decrease in federal research funding - slowing in the expansion of the knowledge base

- Decrease in training - decline in the pool of behavioral scientists

- Decrease in faculty with skills in the field

- Decrease in identification and validation of new drug candidates

and other therapeutic modalities, and by the decline in the number of new product approvals in the face of ever increasing investments in drug discovery. To address these issues, the National Institutes of Health (NIH) launched the Roadmap program (Zerhouni, 2003) and the Food and Drug Administration created the Critical Path Initiatives (2004). Also, in 2006 the U.S. Government General Accountability Office (GAO) published a report detailing factors that slow drug discovery and development. Among those cited was a decline in the ability to translate basic biological discoveries into drugs. In short, the GAO tacitly acknowledged that the emphasis 
on first characterizing molecular mechanisms in the search for new drugs had led to erosion in the expertise ultimately needed to develop chemical leads into viable drug candidates. Among the disciplines that were becoming underrepresented in this regard were the behavioral sciences, and both basic and clinical pharmacology.

The NIH Roadmap is designed to speed the rate of drug discovery and the dissemination of new therapies throughout the medical community. An important component of this undertaking is the Clinical and Translational Science Awards (CTSA's) that fund institutional initiatives in these areas. To be competitive for a CTSA, applicants are encouraged to include service or research components relating to behavioral assessments of health care providers, patients, or laboratory animals to help foster new drug development or the use of old drugs for new purposes. Both the United Kingdom and countries on the European continent have initiated similar programs. In all cases, it is acknowledged that efforts must be made to reestablish the importance of in vivo testing, behavioral observations and analysis in the drug discovery process. Because the expertise necessary for designing such tests is diminishing, funding agencies in the United States, United Kingdom, and Europe are underwriting training programs aimed at exposing biomedical scientists to the fundamentals of whole animal research. In the United States such efforts are exemplified by the Integrative and Organ System Pharmacology courses underwritten by the National Institute of General Medical Sciences (Preusch, 2004).

\section{Conclusion}

As the response to systemically active drugs and other xenobiotics typically involves the interplay of several organs and organs systems, the ability to understand such interactions, and to examine such effects experimentally, is an essential component of the drug discovery process. For a time, however, an obsession with defining drugs primarily in terms of molecular mechanisms led to devaluation of whole animal research and of detailed pharmacological analysis of the effect of test agents on organ system function and behavior. Besides the negative impact this shift in emphasis had on training in the pharmacological and behavioral sciences, it is also acknowledged to have contributed to a decline in the rate of new drug discovery. Given the proven importance of empirical observation in identifying novel drugs, even in the modern era, this consequence is not surprising. The renewed appreciation of the importance of whole animal and organ system research, and the appropriation of funds to reinvigorate training in these areas will help redress the imbalance in preclinical research emphasis in the search for new drugs . The evidence suggests that the most efficient and productive biomedical research programs should include equal measures of studies involving whole animals and organ systems and those aimed at characterizing the biochemical and molecular targets of the drug candidates. Those interested in the behavioral sciences should be heartened by these recent developments as they bode well for the future of this, and related, disciplines. 


\section{$\underline{\text { References }}$}

Enna, S.J. and Williams, M. (2009a). Challenges in the search for drugs to treat central nervous system disorders. Journal of Pharmacology and Experimental Therapeutics 329:329-404.

Enna, S.J. and Williams, M. (2009b). Defining the role of pharmacology in the emerging world of translational research. Advances in Pharmacology 57:10-30.

Food and Drug Administration (2004). Innovation/stagnation. Challenge and opportunity on the critical path to new medical products. www.fda.gov/oe/initiatives/criticalpath/white paper.html

Government Accountability Office (2006). New drug development: Science, business, regulatory, and intellectual property issues cited as hampering drug development efforts. www.gao.gov/htext/d0749.html

Preusch, P.D. (2004). Integrative and organ system pharmacology: A new initiative from the National Institute of General Medical Sciences. Molecular Interventions 4: 72-73.

Zerhouni, F. (2003). The NIH roadmap. Science 302:63-72. 